\title{
Pure Graphogenic Epilepsy - A Rare Form of Reflex Epilepsy
}

\author{
Shaik Afshan Jabeen, A. Haritha Chowdary, K. Rukmini Mridula, Ajith Cherian, P. Rakhee Kumar, \\ Saileja Sarva, Chikkam V.V.S.N. Murty
}

Can J Neurol Sci. 2013; 40: 603-605

A 24-year-old postgraduate student, right handed individual, presented to us in April 2011 with three year duration of jerks involving his hands while writing. The jerks used to start within a few minutes of writing initially in right hand, later spreading to the entire body occasionally resulting in generalized tonic clonic seizures (GTCS) if he continued to write. These symptoms affected his studies and he had difficulty in appearing for exams. There were no other neurological or systemic symptoms. He had a febrile seizure in his early childhood. Birth and developmental history was otherwise unremarkable. There was no family history of epilepsy.

Neurological examination was normal. Neuropsychological examination revealed a normal IQ and normal performance on attention, memory and language tests. Magnetic resonance imaging (MRI) brain (1.5 tesla) was normal.
A diagnosis of writing epilepsy was considered and a short term video EEG recording was performed while the patient was asked to perform various tasks. Each task was provided for five minutes ( $\mathrm{min}$ ) with an interval of five min between two tasks. The interictal awake EEG showed left frontocentral spike and wave discharges with secondary bilateral synchrony. While writing after a latent period of four min, he had myoclonic jerks involving right upper limb which spread to involve other limbs and head within a few seconds. The corresponding EEG revealed spike and wave discharges over left central leads with secondary bilateral synchrony (Figure 1). Spontaneous writing, writing to dictation, writing blindfolded and copying elicited similar response. Myoclonic jerks were elicited on writing with either hand, but prominently with right hand. He had the same frequency and severity of myoclonic jerks whether he wrote in

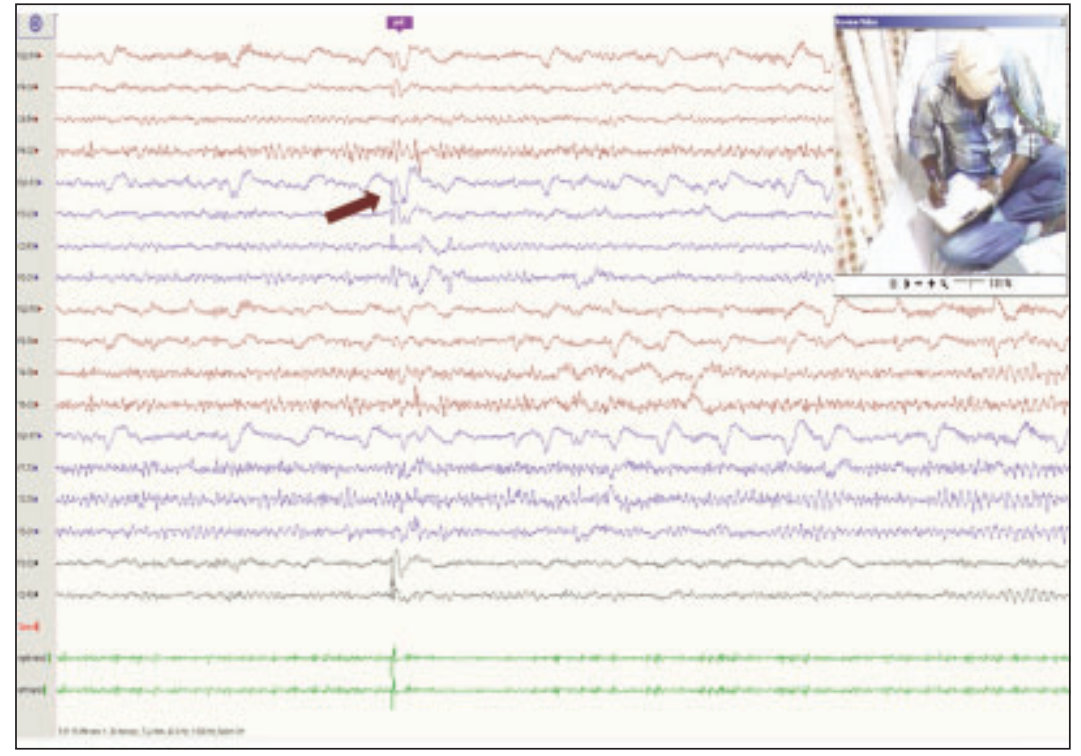

Figure 1: EEG showing spike and wave discharges in left frontocentral leads with secondary bilateral synchrony and EMG electrode documenting corresponding bilateral arm jerks.

From the Department of Neurology (SAJ, AHC, KRM, SS), Department of Radiology (PRK, CVVSNM), Nizam's Institute of Medical Sciences, Hyderabad; Department of Neurology (AC), Trivandrum Medical College, Trivandrum, Kerala, India.

Received November 22, 2012. Final Revisions Submitted February 28, 2013.

Correspondence to: Shaik Afshan Jabeen, Department of Neurology, Nizam’s Institute of Medical Sciences, Punagutta, Hyderabad, India. Email: drjabeennims@gmail.com. 


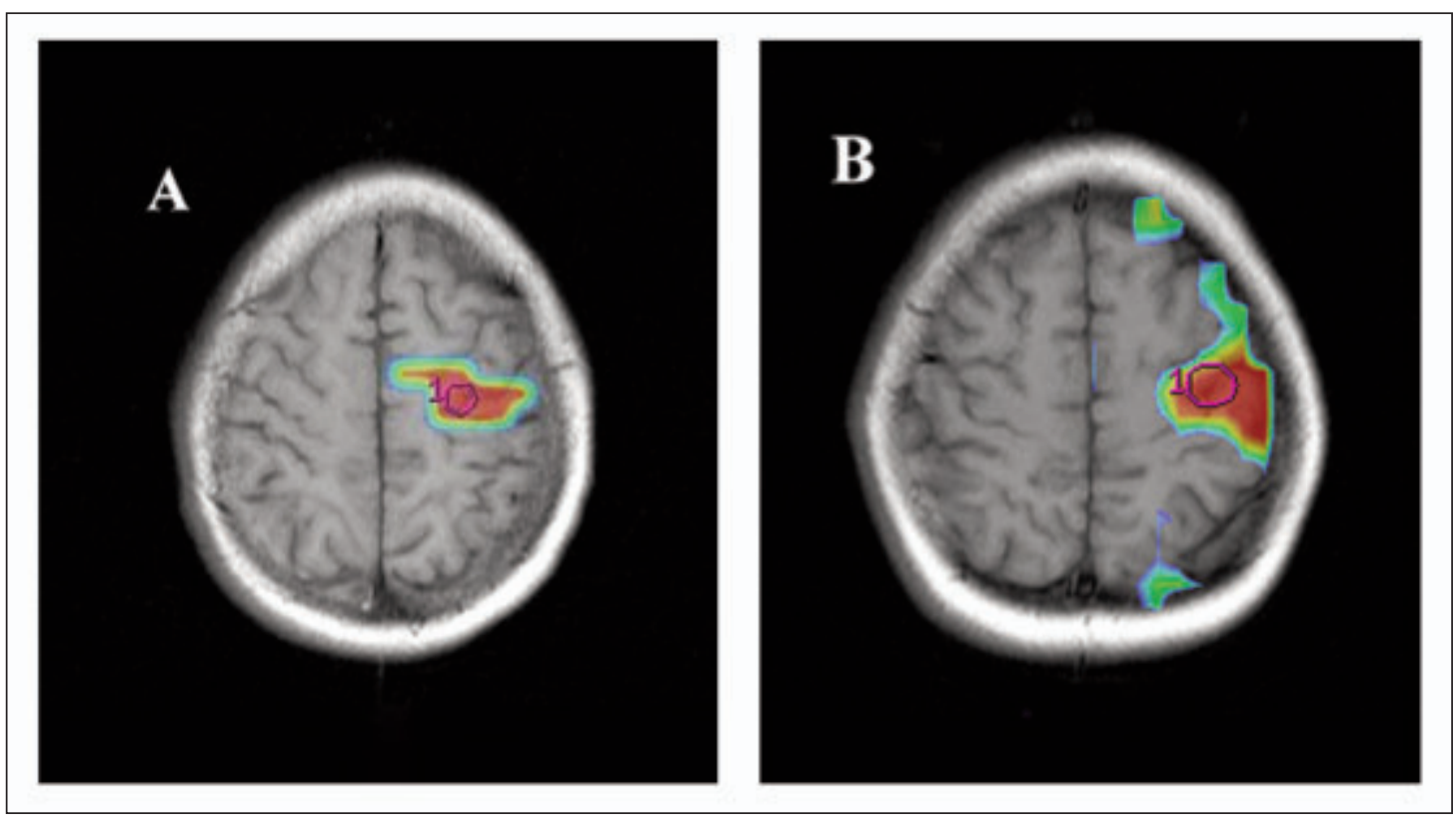

Figure 2: Functional MRI showing similar areas of activation during finger tapping in patient $(A)$ and in control (B).

Telugu (native language) or in English. There was no difference in writing simple or complex sentences. Performing simple and difficult calculations, spontaneous drawing, copying figures, typing on a keyboard with either hand did not evoke any electro clinical activity. Other language tasks like reading or speaking did not induce seizures or epileptiform activity. Hyperventilation and intermittent photic stimulation did not produce any activation.
We performed functional MRI brain in our patient. Functional MRI findings were interesting in our patient, while finger tapping activated similar areas of brain in both patient and age matched control (Figure 2), writing lead to activation of left supplementary motor area and bilateral parasaggital area (left predominant) in the patient and activation of left premotor cortex in control (Figure 3).

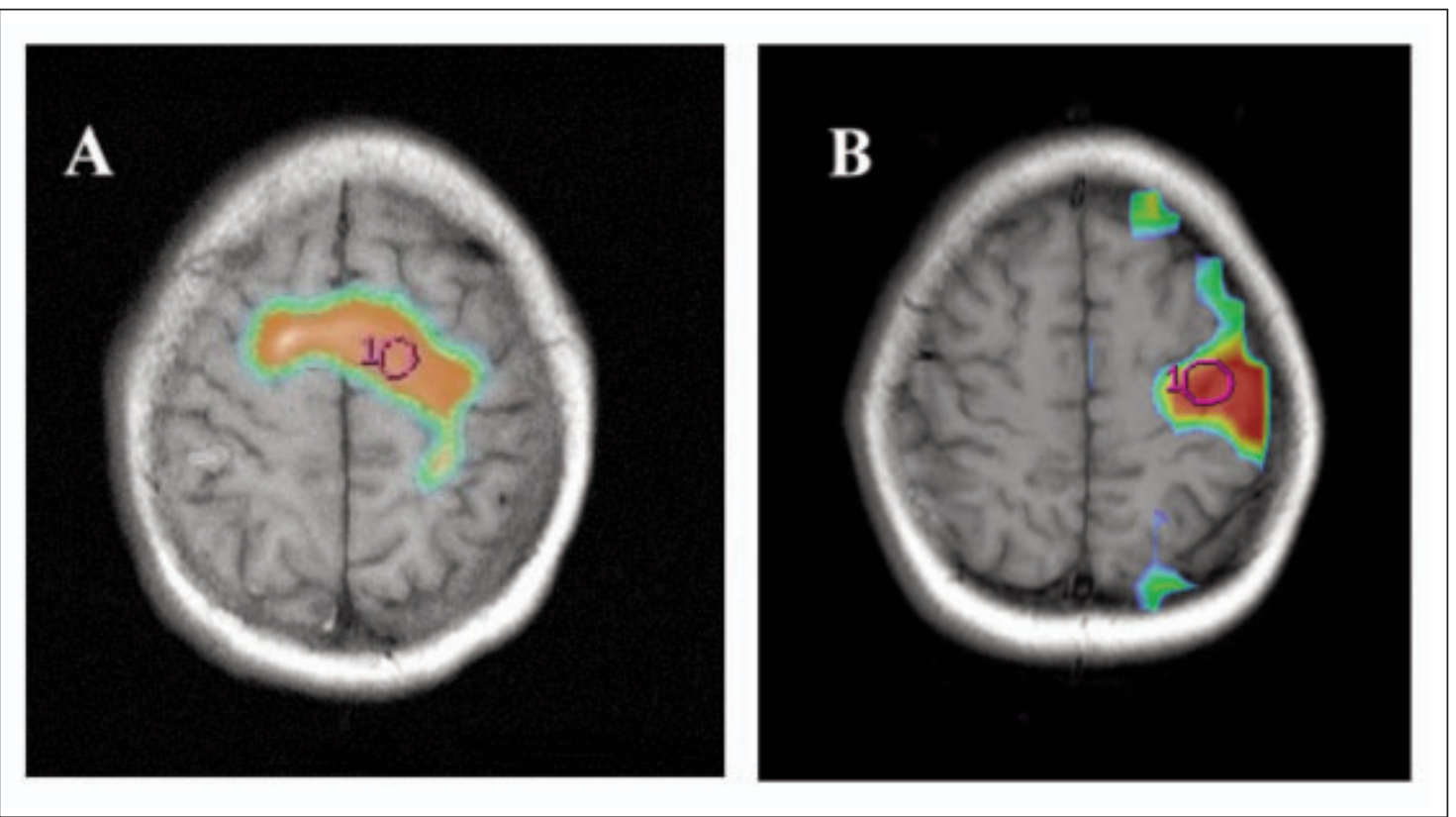

Figure 3: (A) Functional MRI performed on patient during writing paradigm showing left frontal supplementary motor area and bilateral parasagittal area activation. (B) FMRI performed on an age matched control showing activation of left premotor area during writing. 
The patient received sodium valproate at a dose of 1500 $\mathrm{mg}$ /day and clobazam at a dose of $20 \mathrm{mg} /$ day with a significant reduction in jerks and seizures.

In language induced epilepsy, a well known syndrome proposed by Geschwind and Sherwin, different aspects of language function like reading, writing and speaking may precipitate seizures. ${ }^{1}$ Writing epilepsy is a rare reflex syndrome in which seizures are triggered by writing. It is usually associated with seizure precipitation on other language tasks like reading and speaking but pure graphogenic epilepsy is extremely rare. There are only a handful of cases described in the literature. ${ }^{2-4}$

Seizure semiologies described in previous literature on writing epilepsy included varying combinations of myoclonic jerks of upper limbs (especially right hand), speech arrest, and behavioural arrest with altered levels of responsiveness., ${ }^{2,3}$ Our patient had rapid jerks starting in the right hand spreading to involve the left hand and head. He was fully alert and responsive throughout the seizure.

Several EEG abnormalities have been reported with writing epilepsy. Generalized discharges with right centroparietal predominance, ${ }^{3}$ centroparietal diffuse theta activity ${ }^{2}$ and centroparietal spikes and wave discharges ${ }^{4}$ have all been previously described. This is in contrast to reading epilepsy where EEG foci have been found in the left parieto occipital lobe ${ }^{5}$ left temporooccipital lobe ${ }^{6}$ and left supplementary motor cortex. ${ }^{7}$ Ictal EEG in our patient showed frequent transients of spike and wave activity in left central leads with secondary bilateral synchrony probably suggesting a left central focus (Figure 1). A distinct neuroanatomical localization with left central focus in a patient with writing epilepsy has been described by Oshima et al, in 2003. ${ }^{2}$ This is also analogous to the findings by Op de beeck et al where magnetic source imaging of patient with reading epilepsy revealed left central focus (supplementary motor cortex). ${ }^{7}$

Advanced neuroimaging studies were previously performed on patients with writing epilepsy to understand the pathophysiology better. Functional MRI findings in our patient suggest that the hand area is represented normally as evidenced by similar area of activation on finger tapping as with control. But, while writing, extensive left paracentral and supplementary motor cortex were activated suggesting a possible role of left paracentral cortex in pathogenesis of writing induced seizures. A previous case report by Pedro Abreu et al found intense left frontal and supplementary motor area activation. ${ }^{3}$

Pure graphogenic epilepsy is a rare entity. The EEG and functional neuroimaging data in the present case argue in favour of a focal cortical activating mechanism in writing epilepsy in the background of a primary generalized epilepsy syndrome that is typically responsive to treatment with valproic acid plus or minus a benzodiazepine. We propose a distinct neuroanatomical basis with left frontocentral epileptogenic focus in triggering graphogenic seizures, in the setting of presumed primary generalized epilepsy linked diffuse cortical excitability.

\section{REFERENCES}

1. Geschwind N, Sherwin I. Language-induced epilepsy. Arch Neurol. 1967;16(1):25-31.

2. Oshima T, Hirose K, Murakami H, et al. Graphogenic epilepsy: a variant of language-induced epilepsy distinguished from reading- and praxis-induced epilepsy. Seizure. 2003;12(1):56-9.

3. Abreu P, Ribeiro M, Forni A, et al. Writing epilepsy: a neurophysiological, neuropsychological and neuroimaging study. Epilepsy Behav. 2005;6(3):463-6.

4. Tanaka N, Sakurai K, Kamada K, et al. Neuro magnetic source localization of epileptiform activity in patients with graphogenic epilepsy. Epilepsia. 2006;47(11):1963-7.

5. Koutroumanidis M, Koepp MJ, Richardson MP, et al. The variants of reading epilepsy. A clinical and video-EEG study of 17 patients with reading-induced seizures. Brain. 1998;121 (Pt 8): 1409-27.

6. Gavaret M, Guedj E, Koessler L, et al. Reading epilepsy from the dominant temporo-occipital region. J Neurol Neurosurg Psychiatry. 2010;81(7):710-15.

7. Op de beeck M, Legros B, Gaspard N, et al. Supplementary motor cortex involvement in reading epilepsy revealed by magnetic source imaging. Epilepsia. 2011;52(5):e31-4. 